\title{
DETECTION OF MUTATIONS IN SELECTED PROTO-ONCOGENES OF CANINE LYMPHOMA
}

\author{
Bóna, G., Šiviková, K. \\ Department of Biology and Genetics, Institute of Genetics \\ University of Veterinary Medicine and Pharmacy, Košice \\ Slovakia
}

katarina.sivikova@uvlf.sk

\section{ABSTRACT}

Lymphomas belong among the most frequently diagnosed tumours of the haematopoietic system in dogs. The clinical manifestations and genetic and molecular basis of canine lymphoma resembles those of human non-Hodgkin lymphoma and therefore it can serve as a suitable model for the study of this disease. Neoplastic diseases are the consequence of a number of genetic and epigenetic changes in somatic cells. One of such changes are gene mutations that can subsequently cause changes in the activity of proto-oncogenes and tumour suppressor genes. The aim of our study was to detect potential mutations in selected exons of proto-oncogenes in DNA isolated from samples of lymphoma obtained from two donors - a Bernese Mountain Dog and a female mongrel. On the basis of literary data descriptions of human and canine haematopoietic neoplastic diseases, our investigations of potential changes in DNA focused on proto-oncogenes C-KIT - exons 8, 17; NRAS - exons 1, 2;
FLT3 - exons 14, 15 and 20. The investigated samples were amplified by polymerase chain reaction (PCR) and subjected to sequencing. The DNA sequences were compared with reference sequences in the database Ensembl. The comparison of sequences of the C-KIT gene revealed an $\mathrm{A} / \mathrm{G}$ transition at the 35th nucleotide of exon 8 in the mongrel. It involved a synonymous exchange of the nucleotide in the codon that did not cause a change in the amino acid. In the same sample we recorded several point mutations in the intron regions surrounding the exons 14 and 20 of the FLT3 gene. Changes in the intron regions can affect the expression of genes and thus can play an important role in the origin and development of tumours. No genetic mutations were detected in any gene regions of the Bernese Mountain Dog. In the case of the NRAS gene, no changes were observed in any sample collected from the donors.

Key words: canine lymphoma; carcinogenesis; C-KIT; FLT3; NRAS; proto-oncogene 


\section{INTRODUCTION}

Tumour diseases are currently one of the most frequently occurring diseases resulting in high mortality rates [11]. Tumours are considered genetic diseases as their origin and development is associated essentially with changes at the genetic or epigenetic levels in proto-oncogenes and tumour-suppressor genes [15].

Lymphomas are a heterogeneous group of tumour diseases of the immune system blood cells of the lymphoid line. On the basis of pathological findings, lymphomas are divided to two basic categories: non-Hodgkin lymphoma (NHL) and Hodgkin lymphoma (HL). In humans, lymphomas account for $3-4 \%$ of all tumour diseases [9]. However, in dogs they belong among the most frequent types of tumours [14]. The frequency of disease occurrence and similar carcinogenic load on humans and dogs (Canis lupus familiaris) in their common environment are advantageous with respect to investigation of individual types of tumours [5].

The most frequent changes described in relation to lymphomas in humans are translocations in genes MYC, BCL2, BCL6, short mutations/deletions in genes TNFAIP3, PRDM1, EZH2, or amplification of gene JAK2 [1]. Mutations in proto-oncogenes C-KIT, FLT3 and NRAS have been described in association with a number of haematological malignancies including lymphomas. The frequency of these mutations in canine lymphomas has not been specified [13].

The aim of our study was to detect potential genetic changes in selected sections of DNA (exons and/or introns) of the three above mentioned proto-oncogenes.

\section{MATERIALS AND METHODS}

\section{DNA isolation}

Samples of genomic DNA were isolated from dog lymphomas by a column-based method employing QIAGEN DNeasy ${ }^{\circledast}$ Blood and Tissue Kit (Qiagen, Venlo, the Netherlands). Sample 1 was isolated from the lymph node tissue of a 10 years old female mongrel. Sample 2 was obtained from the spleen of a 12 years old Bernese Mountain Dog. The reference DNA sample was isolated from the blood of a healthy Jack Russell Terrier. was used as a positive control.

\section{Selection of primers, PCR and electrophoresis}

Primers were selected by means of software Primer3Plus on the basis of the reference DNA sequences obtained from the database Ensembl. Table 1 shows sequences of the proposed oligonucleotides, access codes of genes and length of the proposed products.

The total volume of the reaction mixture used for PCR was $25 \mu$, which consisted of: 21.1 master-mix (Fire$\left.\mathrm{POL}^{\circledast}\right) ; 1.2 \mu \mathrm{l}$ forward primer; $1.2 \mu \mathrm{l}$ reverse primer; $0.5 \mu \mathrm{l}$ DNA polymerase (FirePOL $\left.{ }^{\circledR}\right) ; 1.0 \mu$ sample. The PCR programme consisted of five steps: initial denaturation of DNA at $95^{\circ} \mathrm{C}$ for $5 \mathrm{~min}$; denaturation of DNA at $95^{\circ} \mathrm{C}$ for $30 \mathrm{~s}$; annealing of primers at $54-56^{\circ} \mathrm{C}$ for $45 \mathrm{~s}$; amplification of the product at $72{ }^{\circ} \mathrm{C}$ for $45 \mathrm{~s}$; final extension at $72^{\circ} \mathrm{C}$ for $45 \mathrm{~s}$. Steps $2-4$ were repeated in $30-35$ cycles. The PCR products were analysed by electrophoresis in agarose gel, stained by intercalation stain GelRed (Biotinum, Hayward, CA, USA) and visualised under UV light (GenoView Smart M-VWR GenoView, Radnor, PA, USA).

Table 1. Primers selected for PCR

\begin{tabular}{|c|c|c|c|c|}
\hline $\begin{array}{c}\text { Gene } \\
\text { (database access code) }\end{array}$ & $\begin{array}{l}\text { Investigated } \\
\text { region }\end{array}$ & $\begin{array}{l}\text { Forward primer } \\
\qquad\left(5^{\prime} \rightarrow 3^{\prime}\right)\end{array}$ & $\begin{array}{l}\text { Reverse primer } \\
\qquad\left(5^{\prime} \rightarrow 3^{\prime}\right)\end{array}$ & $\begin{array}{l}\text { Product length } \\
\text { (bp) }\end{array}$ \\
\hline \multirow{2}{*}{$\begin{array}{l}\text { C-KIT } \\
\text { (ENSCAFG00000002065.4) }\end{array}$} & exon 8 & TTCACTCACTGGTTCCGATGC & ССTATCTGAAGTTCAACTACC & $340 \mathrm{bp}$ \\
\hline & exon 17 & GTGACATAGCAGCATTCTCG & GCATGGTATCTCAAAGGTAGG & $333 \mathrm{bp}$ \\
\hline \multirow{2}{*}{$\begin{array}{l}\text { FLT3 } \\
\text { (ENSCAFG00000006716) }\end{array}$} & exons 14,15 & GGCCCTTCCCTTTCATCCAA & CCATGCCTCCCATTTTTGTGC & 572 bp \\
\hline & exon 20 & GGTGGTGGCCCAGTAAAGAT & GAAGCTTTTGGATGCTGCCA & $388 \mathrm{bp}$ \\
\hline \multirow{2}{*}{$\begin{array}{l}\text { NRAS } \\
\text { (ENSCAFG00000009532) }\end{array}$} & exon 1 & TTCACGTGTATGCAGCCGAT & TACTCCTGAATGCAGCACCC & 379 bp \\
\hline & exon 2 & TGGAAAGAACTCAGCTAGGAGC & TCCCTAATGCGGTATCCTCA & $387 \mathrm{bp}$ \\
\hline
\end{tabular}




\section{DNA sequencing}

The DNA sequencing was done by an ABI PRISM 3100-Avant Genetic Analyser (Applied Bio-systems, Waltham, MA, USA) in the Laboratory of Biomedicinal Microbiology and Immunology of the University of Veterinary Medicine and Pharmacy (UVMP) in Košice. The sequences obtained from samples were compared with reference sequences using algorithms BLAST ("Basic Local Alignment Search Tool“): BLASTN and BLASTX, at web interface Ensembl.

\section{RESULTS}

From the C-KIT gene we obtained sufficient amounts of amplified sections of exons 8 and 17 from both samples (Fig. 1a). In sample 1 (mongrel) we detected a nucleotide exchange - transition G/A (Fig. 1b) in exon 8 at the 67290 nucleotide of C-KIT gene (ENSCAFG00000002065.4) (Fig. 1c). In the database Ensembl this mutation is described as single-nucleotide polymorphism (SNP) (under identification number rs22299980). No mutation in exon 17 was observed in the mongrel. In the Bernese Mountain Dog (sample 2) no mutations were detected in either of two exons.

From the FLT3 gene we obtained from both samples sufficient amount of amplified sections that included exons 14-15 and 20 and the surrounding intron regions (Fig. 2a). In sample 1 (mongrel) we detected several changes in intron regions close to exons 14 and 15 (Fig. 2d); in 34 nt position in front of exon 14-transversion A/C (Fig. 2b), and in $263 \mathrm{nt}$ position, between exons 14 and 15 - transition G/A (Fig. 2c).

When comparing sample 1 with the reference sequence (Fig. 3a), we detected a transition C/T (Fig. 3b) in the intron region behind exon 20 , in 242 nt position. In the sec-

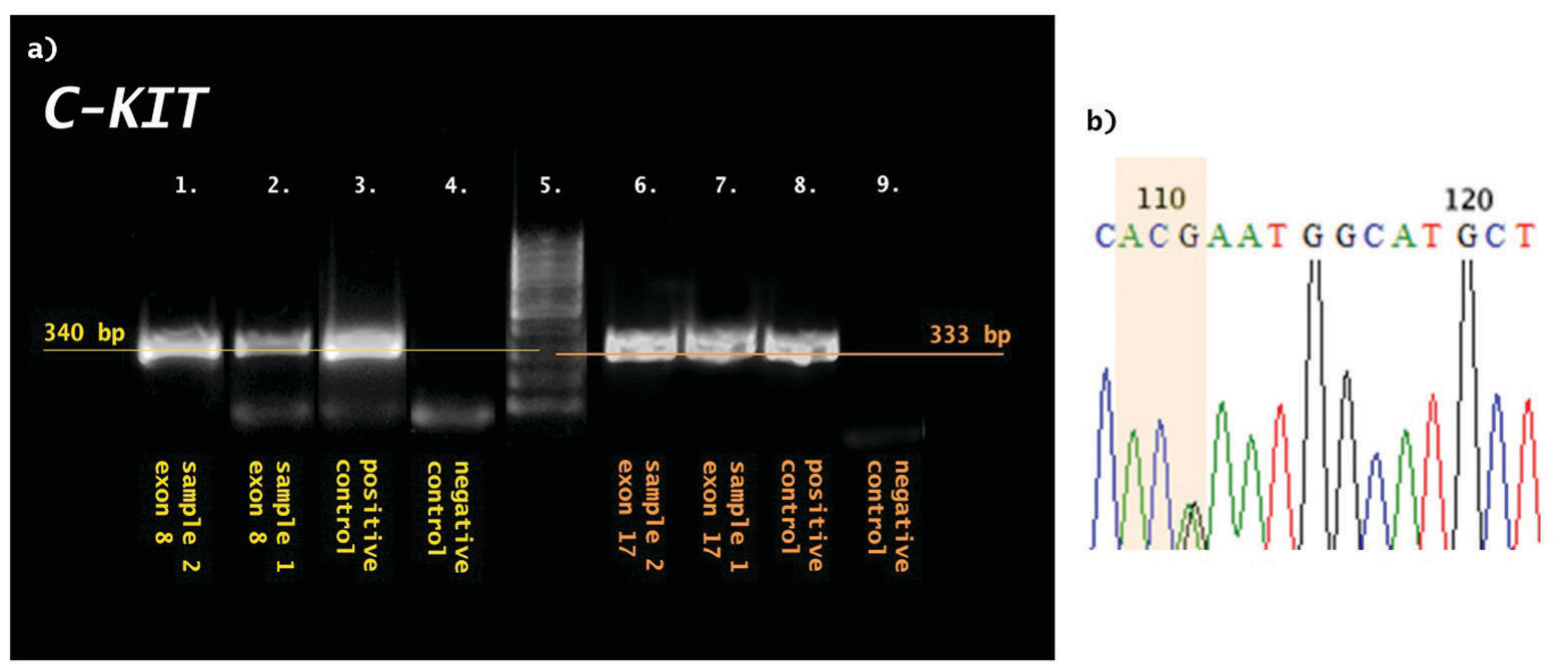

c)

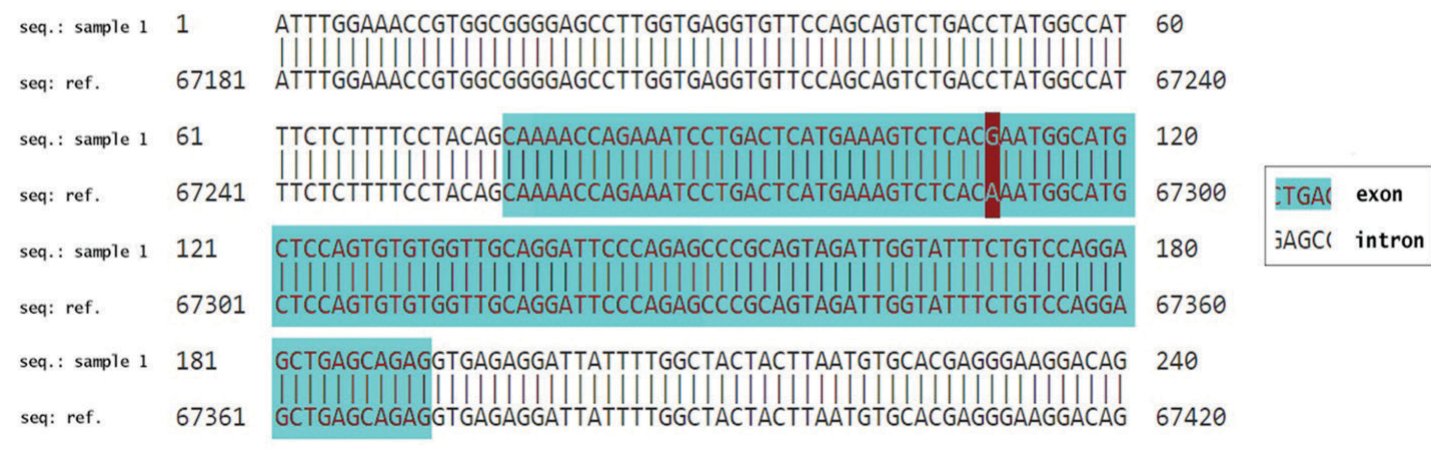

Fig. 1. Electrophoretic evaluation of the amplification of C-KIT gene sections - exons 8 and 17 - the 100 bp DNA ladder is located in the central (5th) column. Numbers of bp shown on the sides of the picture give the exact number of base pairs in the proposed section. DNA sequence with highlighted nucleotide exchange - G/A transition in exon 8 at the 67290 nucleotide of C-KIT gene. Comparison of DNA sequence of sample 1 (mongrel) with reference sequence; nucleotide exchange is located at the 33rd nucleotide of exon 8 


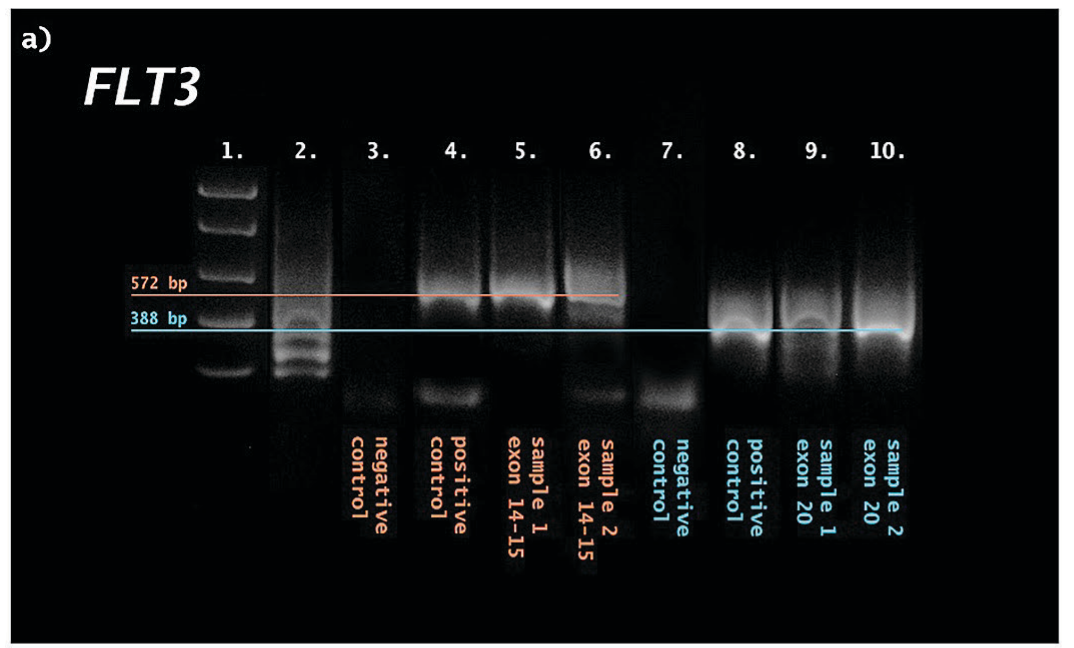

b) 33

42

AAC A GT AAAAAT

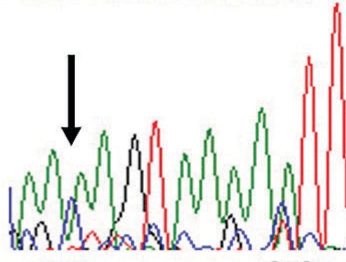

c)

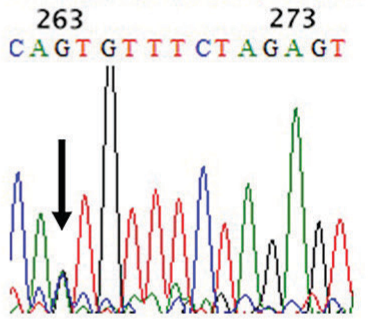

d) seq.: sample 11

$$
\begin{array}{ll}
\text { seq.: sample } 1 & 1 \\
\text { seq.: ref. } & 51770 \\
\text { seq.: sample 1 } & 61 \\
\text { seq.: ref. } & 51830 \\
\text { seq.: sample 1 } & 121 \\
\text { seq.: ref. } & 51890 \\
\text { seq.: sample 1 } & 181 \\
\text { seq.: ref. } & 51950 \\
\text { seq.: sample 1 } & 241 \\
\text { seq.: ref. } & 52010
\end{array}
$$

TTATTTGTCACAAGTACAAAAAGGTAAAAGCAACAGTAAAAATTCATCCCCATTTCCCAT 60

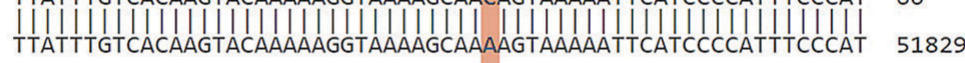
TTCTGAGGGACTGCCTGTTGCTAATGGACCCATTATTTCACTTCTGAAACAATTTAGGTA 120 TTCTGAGGGCTGCCTGTGCTAATGGACCCATTATTTCACTTCTGAGCAATTAGGTA 51889

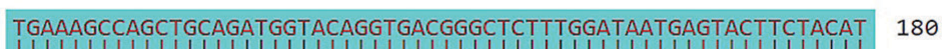

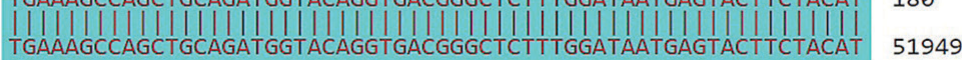
TGATTTCAGAGAATATGAATATGATCTCAAATGGGAGTTTCCCAGAGAAAATTTAGAATT 240

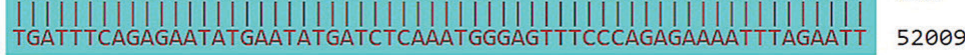

TGGTAAGACTCGAATGGTCCCAGTGTTTCTAGAGTATTTCCTTTCTGTTGGGAATCTCT 300 TGGTAAGACTCGAATGGTCCCAATGTTCTAGAGTATTTCCTTTCTGTGGGAATCTCT 52069

Fig. 2. a) Electrophoretic determination of amplification of gene FLT3 sections - exons 14, 15 and 20. The first column shows DNA ladder with divisions $100 \mathrm{bp}, 400 \mathrm{bp}, 800 \mathrm{bp}, 2000 \mathrm{bp}$ and $5000 \mathrm{bp}$. The $100 \mathrm{bp}$ DNA ladder is in the second column. Coloured numbers of base pairs on the left of this figure correspond to the exact number of bp of the proposed product; b) DNA sequence with highlighted nucleotide exchange $-\mathrm{C} / \mathrm{A}$ transversion in FLT3 gene, position 34; c) SDNA sequence with highlighted nucleotide exchange - G/A transition in FLT3 gene, position 263; d) Comparison of DNA sequence of sample 1 (mongrel) with reference sequence - gene FLT3, exon 14

a) seq.: sample 11

seq.: ref. 63818

seq.: sample 161

seq.: ref. 63878

seq.: sample 1121

seq.: ref. 63938

seq. : sample 1181

seq.: ref. 63998

seq. : sample 1241

seq.: ref. 64058

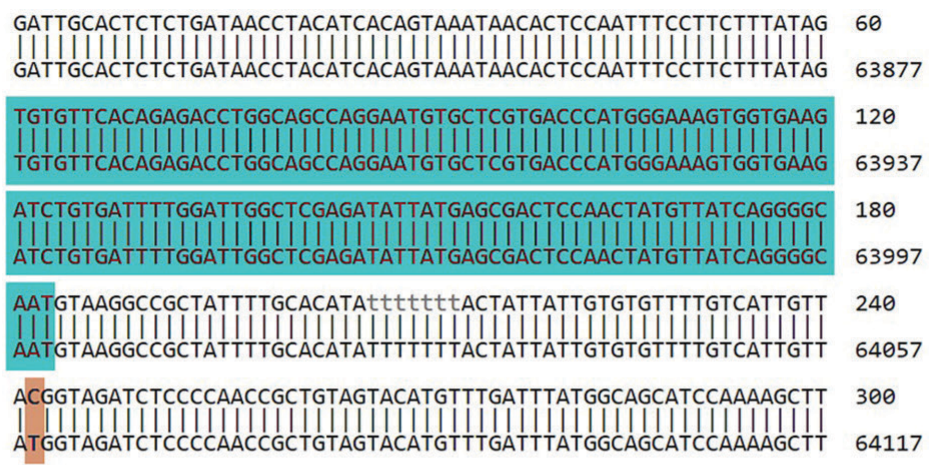

3937

\section{iTGAl exon}

jAGCl intron

(b)

b)

234

244

I CAT T G T T ACG GT A

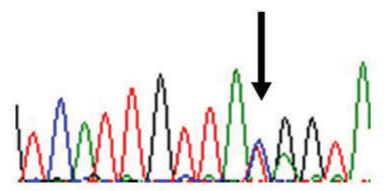

Fig. 3. Comparison of DNA sequence of sample 1 (mongrel) with the reference sequence - FLT3 gene, exon 20 DNA sequence with arrow indicating nucleotide exchange - C/A transversion, FLT3 gene, position 242 
ond sample no exchange in DNA sequence was observed either in exons 14, 15 and 20, or their surroundings.

From the NRAS gene we obtained from both samples sufficient amounts of amplified sections that included exons 1 and 2. No changes in the selected sections were observed in any of the two samples.

\section{DISCUSSION}

Lymphomas belong among the most frequent tumour diseases in dogs. The selection of proto-oncogenes in our samples from the mongrel and Bernese Mountain Dog was based on the data from the literature describing mutations in proto-oncogenes C-KIT, FLT3 and NRAS.

Proto-oncogene C-KIT encodes a tyrosine kinase receptor that is essential for normal haematopoiesis, pigmentation, fertility and other functions. The canine KIT protein is composed of 975 amino acids. Our study showed no mutations in selected exons (8 and 17) of the C-KIT gene resulting in the exchange of amino acids in this protein. Synonymous exchange, A/G transition, was recorded in 425th codon, nucleotide 67290, encoding the amino acid threonine. This involves a synonymous variant, i.e. a change that has already been observed in dogs [13] and does not affect the significance of the codon. Our results agree with those of Giantin et al. [3] according to which the occurrence of mutations in canine lymphoma C-KIT gene is rare.

In relation to the damage in the C-KIT gene, cases have been described of acute myeloid leukaemia [13] and adipose cell tumours [6]. Lond on et al. [6] reported the occurrence of intragene tandem duplications in exons 11 and 12 associated with tumour diseases of mastocytes. In dogs with acute myeloid leukaemia diagnosis, Usher et al. [13] found silent mutations in exon 8 , codon 425 , and a number of point mutations resulting in changes in AMK sequence in the exon 17. In the aggressive canine T-cell lymphomas, Giantin et al. [3] observed increased expression of C-KIT oncogene in comparison with less aggressive forms of the disease, while in B-cell lymphomas expression of this protein was reduced.

FLT3 is a tyrosine-kinase receptor included in the same family of receptors as KIT. It plays an important role in normal development and proliferation of stem and progenitor cells of the haemapoietic system [10]. In the sample obtained from the Bernese Mountain Dog in our study we did not record any exchange in the investigated exons. Several point mutations were detected in FLT3 gene in the sample from the mongrel, namely three exchanges in the intron regions in the surroundings of exons $14-15$ and exon 20. Transversion A/C in 51803 nt position has not yet been described in the database Ensembl. Transition G/A in position $52033 \mathrm{nt}$ and transition C/T in position $64059 \mathrm{nt}$ of the gene have been described in the database Ensembl as intron variants, i.e. single nucleotide polymorphisms. Intron sequences have been considered in the past as useless DNA, however, the recent results of molecular genetics indicate that intron SNP may affect initiation, elongation and termination of transcription, polyadenylation, export of mRNA from cell nucleus and the stability of the relevant mRNA [4].

U sher et al. [13], described intragene tandem duplications in exons 14 and 15 of gene FLT3 in 3 out of 57 cases of canine acute myeloid leukaemia. In this study, the authors identified also point mutation changing importance of a codon in exon 20. Other authors [12] reported the occurrence of intragene tandem duplications and increased values of mRNA of the gene in 2 out of 7 cases.

The family of RAS genes includes genes NRAS, HRAS and KRAS, that encode small intracellular proteins (189 amino acids) bound to cellular membrane. The primary structure of protein RAS is conserved in a large number of mammals. These genes exhibit GTPase activity, partaking in a number of signalling pathways. They mediate signals affecting proliferation, differentiation and apoptosis of cells [8]. Mutations in the proto-oncogenes of RAS family have been frequently described in relation to various neoplasms in rodents and man. Activating point mutations have been observed particularly in exons 1 and 2 of NRAS gene, namely in codons 12, 13 and 61 [2].

In our study we observed no change in genetic code in comparison with reference sequence, which is in agreement with observations of Mayr et al. [7] who reported that activation of proto-oncogene NRAS in canine lymphoma is rare.

\section{CONCLUSIONS}

The results of our study allowed us to conclude that proto-oncogenes C-KIT and NRAS probably did not play an important role in the process of lymphomagenesis in dogs. 
We identified a silent mutation caused by nucleotide exchange in exon 8 of gene C-KIT and SNP in intron regions in the surroundings of exons 14, 15 and 20 of gene FLT3. SNP can be found in various regions of genomic DNA and occur relatively frequently in dogs. SNP in introns may cause their erroneous expression, either due to aberrant splicing or error in the regulation region of the gene and thus affect the process of carcinogenesis.

More information may be obtained by the study of the changes in gene expression or epigenetic changes, particularly in the Bernese Mountain Dog, since our study dog was in an advanced stage of the disease.

\section{ACKNOWLEDGEMENT}

This study was supported by the projects VEGA 1/0043/15 and VEGA 1/0176 MŠ SR.

\section{REFERENCES}

1. Devita, V.T., Lawrence, T.S., Rosenberg, S. A., 2015: Cancer: Principles and Practice of Oncology, 10th edn., Lippincott Williams and Wilkins, $2234 \mathrm{pp}$.

2. Edwards, M.D., Pazzi, K. A., Gumerlock, P.H., 1993: cNras is activated infrequently in canine malignant lymphoma. Toxicol. Pathol., 21, 288-291.

3. Giantin, M., Aresu, L., Aricò, A., Gelain, M.E., Riondato, F., Comazzi. S. et al., 2013: Evaluation of tyrosine-kinase receptor c-kit mutations, mRNA and protein expression in canine lymphoma: Might c-kit represent a therapeutic target? Vet. Immunol. Immunopathol., 154, 153-159.

4. Chorev, M., Carmel, L., 2012: The function of introns. Frontiers in genetics, $3,1-15$.
5. Ito, D., Frantz, A.M., Modiano, J.F., 2014: Canine lymphoma as a comparative model for human non-Hodgkin lymphoma: recent progress and applications. Vet. Immunol. Immunopathol., 159, 192-201.

6. London, C.A., Galli, S.J., Yuuki, T., Hu, Z.Q., Helfand, S. C., Geissler, E. N., 1999: Spontaneous canine mast cell tumors express tandem duplications in the proto-oncogene ckit. Exp. Hematol., 27, 689-697.

7. Mayr, B., Winkler, G., Schaffner, G., Reifinger, M., Brem, G., 2002: N-ras mutation in a feline lymphoma. Low frequency of $\mathrm{N}$-ras mutations in a series of feline, canine and bovine lymphomas. Vet. J., 163, 326-328.

8. Richter, A., Escobar, H.M., Gunther, K., Soller, J.T., Winkler, S., Nolte, I. et al., 2005: RAS gene hot-spot mutations in canine neoplasias. J. Hered., 96, 764-765.

9. Roman, E., Smith, A. G., 2011: Epidemiology of lymphomas. Histopathology, 58, 4-14.

10. Small, D., 2006: FLT3 mutations: biology and treatment. ASH Education Program Book, 2006, 178-184.

11. Stewart, B. W., Wild, C. P., 2014: World Cancer Report 2014. Lyon, International Agency for Research on Cancer, 630 pp.

12. Suter, S. E., Small, G. W., Seiser, E. L., Thomas, R., Breen, M., Richards, K. L., 2011: FLT3 mutations in canine acute lymphocytic leukemia. BMC cancer, 11, 38 .

13. Usher, S. G., Radford, A.D., Villiers, E. J., Blackwood, L., 2009: RAS, FLT3, and C-KIT mutations in immunophenotyped canine leukemias. Exp. Hematol., 37, 65-77.

14. Valli, E. V., San Myint, M., Barthel, A., Bienzle, D., Caswell, J., Colbatzky, F. et al., 2011: Classification of canine malignant lymphomas according to the World Health Organization criteria. Vet. Pathol., 48, 198-211.

15. Weinberg, R., 2013: The Biology of Cancer. Garland Science, $876 \mathrm{pp}$.

Received June 5, 2017

Accepted September 28, 2017 